\title{
Approximate Medial Axis as a Voronoi Subcomplex *
}

\author{
Tamal K. Dey \\ Dept. of CIS \\ Ohio State University \\ Columbus, OH 43210, USA \\ tamaldey@cis.ohio-state.edu
}

\author{
Wulue Zhao \\ Dept. of CIS \\ Ohio State University \\ Columbus, OH 43210, USA \\ zhaow@cis.ohio-state.edu
}

\begin{abstract}
Medial axis as a compact representation of shapes has evolved as an essential geometric structure in a number of applications involving 3D geometric shapes. Since exact computation of the medial axis is difficult in general, efforts continue to approximate them. One line of research considers the point cloud representation of the boundary surface of a solid and then attempts to compute an approximate medial axis from this point sample. It is known that the Voronoi vertices converge to the medial axis for a curve in $2 \mathrm{D}$ as the sample density approaches infinity. Unfortunately, the same is not true in 3D. Recently, it is discovered that a subset of Voronoi vertices called poles converge to the medial axis in $3 \mathrm{D}$. However, in practice, a continuous approximation as opposed to a discrete one is sought.

Recently few algorithms have been proposed which use the Voronoi diagram and its derivatives to compute this continuous approximation. These algorithms are scale or density dependent. Most of them do not have convergence guarantees, and one of them computes it indirectly from the power diagram of the poles. Recently we proposed a new algorithm that approximates the medial axis straight from the Voronoi diagram in a scale and density independent manner with convergence guarantees. In this paper we present several experimental results with this algorithm that support our theoretical claims and also show its effectiveness on practical data sets.
\end{abstract}

\section{Categories and Subject Descriptors}

I.3.5 [Computer Graphics]: Computational Geometry and Object Modeling; I.4 [Image Processing and Computer Vision]: Reconstruction,Image Representation

\section{General Terms}

Algorithms, Experimentation, Theory, Design

\footnotetext{
*This work is partially supported by NSF under grants CCR-9988216 and DMS-0138456.
}

\section{Keywords}

Medial axis, Point cloud, Voronoi diagram

\section{INTRODUCTION}

Medial axis of a shape provides a compact representation of its features and their connectivity. As a result, researchers have discovered and are still exploring its use in a number of applications ranging over image processing [22], computer vision [6, 23], solid modeling [19, 20, 26, 27], mesh generation $[24,25]$ and many others $[18,21,29]$. Medial axis is defined when the shape is embedded in an Euclidean space and is endowed with a distance function. In this paper we are concerned with the medial axis of surfaces embedded in three dimensions. Informally, the medial axis of a surface in $3 \mathrm{D}$ is the set of all points that have more than one closest point on the surface. They are often called the medial axis transform or MAT in short for the three dimensional solid bounded with the surface.

Driven by the need of applications, a handful of researchers have focused on the computational aspects of the medial axis. Exact computation of the medial axis is hard in general. Culver, Keyser and Manocha [10] and Hoffman [20] have given algorithms for computing the exact medial axis for some special class of shapes. Even these algorithms have to deal with the numerical instability associated with the medial axis computations. This instability can be attributed in part to the fact that the medial axis is sensitive to tiny changes in shape [17,30]. Consequently, efforts have been made to approximate the medial axis. For polyhedral input Etzion and Rappoport [16] suggest a method for medial axis approximation using an octree subdivision of space. Another scheme considered by many uses a set of sample points on the shape boundary and then approximates the medial axis with the Voronoi diagram of these points $[3,5,4,9,24$, $28,29]$.

We follow the Voronoi diagram approach. It is apt for point clouds that are being used for geometric modeling over a wide range of applications with rapidly advancing sampling technology. It is known that the Voronoi vertices approximate the medial axis of a curve in $2 \mathrm{D}$. In fact, Brandt [8] showed that if the sample density approaches infinity, the Voronoi vertices in this case converge to the medial axis. Unfortunately, the same is not true in three dimensions. Amenta and Bern observe that some Voronoi vertices can come close to the surface [1]. These are the centers of the flat tetrahedra called 'slivers'. In the context of surface reconstruction, they also observe that some Voronoi vertices called 'poles' remain far from the surface. These poles are 
the farthest Voronoi vertices from the sample points in their Voronoi cells. Boissonnat and Cazals [7] and Amenta, Choi and Kolluri [3] show that the poles indeed lie close to the medial axis and converge to it as sample density approaches infinity.

For most practical applications, a continuous approximation of the medial axis is required rather than a set of discrete set of points even if they lie close to the medial axis. In 2D, Brandt and Algazi [9] achieve this by retaining a subset of Voronoi edges incident to the Voronoi vertices. In 3D since poles lie close to the medial axis, Amenta, Choi and Kolluri [3] design an algorithm that connects them with a cell complex. They consider the Delaunay balls centering the poles and then compute the medial axis of the boundary of the union of these balls. Since this union approximates the original object whose boundary is sampled, the computed medial axis approximates the original one. Although theoretically sound, this method requires two Voronoi diagram computations and produces noisy medial axis in some cases.

In this paper we experiment with an algorithm that approximates the medial axis directly from the Voronoi diagram. This approach does not pay any special attention to poles, but rather computes a subcomplex from the Voronoi diagram that lies close to the medial axis and converges to it as sampling density approaches infinity. Approximating the medial axis from the Voronoi diagram in 3D has been attempted in the past. Attali and Montanvert [5] and Attali and Lachaud [4] compute the subset of the Voronoi facets, edges and vertices that reside 'inside' the surface and then prune some Voronoi vertices and their incident elements according to some angle and length criteria. Although the strategy achieves good results in many cases, the pruning in [5] is insufficient as we argue later and the pruning in [4] is scale dependent and more seriously depends on the sampling density. Consequently, one needs to fine tune the pruning parameters individually for each data set and it is not clear if these strategies are apt for a data where the density varies in different parts of the shape.

The algorithm we explore also uses two criteria to select the Voronoi facets from the Voronoi diagram. But, unlike [4], these two criteria are scale and density independent. The Delaunay edges are filtered from the Delaunay triangulation of the sample points and then their dual Voronoi facets are output as an approximate medial axis. The filtration is such that the thresholds used for two criteria remain fixed over data sets of different densities. Thus, there is no need for fine tuning the parameter values. The convergence guarantee of this algorithm is proved in a companion paper [13]. We present several experimental results with this new algorithm. We also perform some experiments on two strategies of simplifying the medial axis.

The rest of the paper is organized as follows. Section 2 contains preliminaries and definitions that we use later. Section 3 details the two conditions that we use to filter the Delaunay edges. Section 4 describes the algorithm and the experimental results. Section 5 illustrates experimental results on simplifying the approximated medial axis. Section 6 contains concluding remarks.

\section{SURFACE SAMPLES}

Let $P$ be a point sample from a smooth compact surface $S \subset \mathbb{R}^{3}$ without boundary. A ball is called medial if it meets $S$ only tangentially in at least two points. The medial axis
$M$ of $S$ is defined as the closure of the set of centers of all medial balls. Each point on $S$ has two medial balls, one touching it from outside and the other touching it from inside. It follows that the line going through a point $p \in S$ and the centers of its medial balls is normal to $S$ at $p$. See Figure 1 for an illustration in 2D.

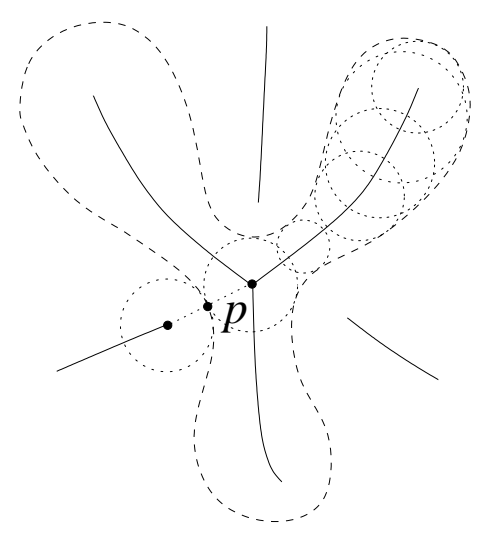

Figure 1: A curve and its medial axis in 2D.

Obviously, a sample $P$ needs to be dense enough to contain information about the features of $S$. Following [1] we define the local feature size $f()$ as a function $f: S \rightarrow \mathbb{R}$ where $f(x)$ is the distance of $x \in S$ to the medial axis $M$. Intuitively, $f()$ measures how complicated $S$ is locally. A sample is an $\varepsilon$-sample if each point $x \in S$ has a sample point within $\varepsilon f(x)$ distance. We have observed that $\varepsilon \leq 0.4$ is sufficient for approximating $S$ with a piecewise linear surface in practice [11].

The Voronoi diagram and its dual, the Delaunay triangulation, plays a key role in capturing information about shapes from a sufficiently dense sample. This observation has led to a number of algorithms for the related problem of surface reconstruction which exploit the structures of these diagrams $[1,2,7,11,14,15]$. The Voronoi diagram $V_{P}$ for a point set $P \in \mathbb{R}^{3}$ is a cell complex consisting of Voronoi cells $\left\{V_{p}\right\}_{p \in P}$ and their facets, edges and vertices, where $V_{p}=\left\{x \in \mathbb{R}^{3} \mid\|p-x\| \leq\|q-x\|, \forall q \in P\right\}$. The dual complex, $D_{P}$, called the Delaunay triangulation of $P$, consists of Delaunay tetrahedra and their incident triangles, edges and vertices. A Delaunay tetrahedron is dual to a Voronoi vertex, a Delaunay triangle is dual to a Voronoi edge, a Delaunay edge is dual to a Voronoi facet and a Delaunay vertex is dual to a Voronoi cell. It is an important result proved in [1] that the Voronoi cells are elongated along the normal direction to the surface at the sample points if the sample is sufficiently dense. The definition of poles as borrowed from [1] plays an important role in approximating these normals.

Definition 1. The pole $p^{+}$of a sample point $p$ is the farthest Voronoi vertex in the Voronoi cell $V_{p}$. If $V_{p}$ is unbounded, $p^{+}$is taken at infinity. The vector $\mathbf{v}_{p}=p^{+}-p$ is called the pole vector for $p$ and its direction is taken as the average of all directions of infinite edges in case $V_{p}$ is unbounded, see Figure 2.

It is proved in [1] that the pole vector $\mathbf{v}_{p}$ approximates the normal $\mathbf{n}_{p}$ to the surface $S$ at $p$ up to orientation.

DEFINITION 2. The tangent polygon for a sample point is 


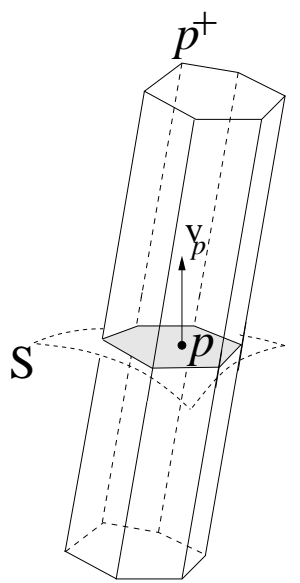

(a)

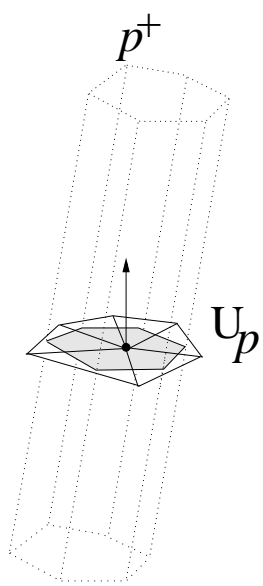

(b)
Figure 2: A Voronoi cell $V_{p}$. The corresponding pole, pole vector, tangent polygon (a), and the umbrella (b).

defined as the polygon in which the plane through $p$ with $\mathbf{v}_{p}$ as normal intersects $V_{p}$. See Figure 2(a) for an illustration.

Since $\mathbf{v}_{p}$ approximates $\mathbf{n}_{p}$, the tangent polygon approximates the tangent plane at $p$ restricted within $V_{p}$. We define a dual structure to the tangent polygon from the Delaunay triangulation $D_{P}$.

DEFINITION 3. The umbrella $\mathrm{U}_{p}$ for a sample point $p$ is defined as the topological disc made by the Delaunay triangles incident to $p$ that are dual to the Voronoi edges intersected by the tangent polygon, see Figure 2(b).

In what follows we will denote the angles between two vectors $\mathbf{u}$ and $\mathbf{v}$ by $\angle \mathbf{u}, \mathbf{v}$ where the angle is measured as the acute one between the lines supporting $\mathbf{u}$ and $\mathbf{v}$.

\section{FILTER CONDITIONS}

Our aim is to approximate the medial axis $M$ with a subset of Voronoi facets and their incident edges and vertices. We choose these Voronoi facets as a dual of a set of selected Delaunay edges. This means we need some criteria to filter these Delaunay edges from $D_{P}$. Let us examine a medial ball $B$ closely to determine the Delaunay edges to be filtered. Consider Figure 3. The segment $p q$ makes an angle $\theta$ with the tangent planes at $p$ and $q$ where the medial ball touches the surface $S$. If $B$ touches $S$ in more than two points, let $p$ and $q$ be such that the angle $\theta$ is maximum. We associate each medial axis point $m$ with such an angle $\theta$, which we call the medial angle of $m$. We approximate each medial axis point with the medial angle $\theta$ greater than a threshold with an Angle condition. For the rest of the medial axis points we apply a Ratio condition.

\subsection{Angle condition}

The approximation of the medial angle $\theta$ for a medial axis point requires approximation to the tangent planes at that points where the medial ball touch the surface. It is known that a set of Delaunay triangles lie close and flat to the sampled surface $S$ if $P$ is dense, see [2] for a precise statement.

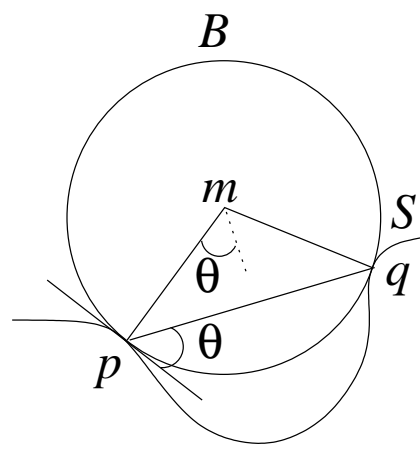

Figure 3: A medial axis point $m$, its medial angle $\theta$ and the corresponding medial ball.

In particular, the triangles in the umbrellas necessarily lie close to $S$ with their normals almost matching those of $S$ at their vertices.

Therefore, we take umbrella triangles in $\mathrm{U}_{p}$ for approximating the tangent plane at a sample point $p$ and determine all Delaunay edges $p q$ that make relatively large angle with this tangent plane. To compute this we measure the angle $\angle \mathbf{n}_{\sigma}, \mathbf{t}_{p q}$ between the unit vector $\mathbf{t}_{p q}$ from $p$ to $q$ and the normal $\mathbf{n}_{\sigma}$ to a triangle $\sigma$ in $\mathrm{U}_{p}$. We select those edges that make this angle less than a threshold angle $\frac{\pi}{2}-\theta$ for all umbrella triangles in $\mathrm{U}_{p}$ for $0<\theta<\frac{\pi}{2}$, see Figure 4. This means a selected edge $p q$ satisfies:

Angle condition $[\theta]: \max _{\sigma \in \mathrm{U}_{p}} \angle \mathbf{n}_{\sigma}, \mathbf{t}_{p q}<\frac{\pi}{2}-\theta$.

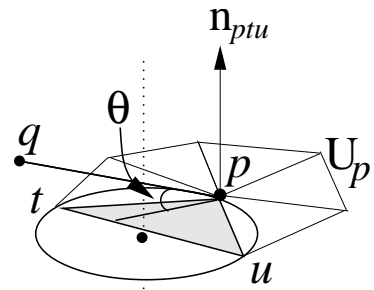

Figure 4: The angle $\theta$ for the Angle condition $[\theta]$. The Voronoi edge shown with the dotted line is parallel to the normal of the shaded triangle.

\subsubsection{Experiments.}

First, we experiment with the 'only Angle condition' to select the the output facets, i.e., only those Voronoi facets are output whose dual Delaunay edges satisfy the Angle Condition $[\theta]$. We varied the value of $\theta$ in order to get a good approximation to the medial axis. As expected, larger value of $\theta$ produces less facets in the output resulting in undesirable 'holes', see the rightmost picture of 3 HOLE data in Figure 5. On the other hand, smaller values of $\theta$ generate too many facets resulting in undesirable 'spikes', see the leftmost picture for the 3 HoLE data in Figure 5. For each model shown in Figure 5 we attempted to determine a value of $\theta$ for which we can obtain an approximation as good as possible. The second row of Figure 5 shows the output of this experiment. The major drawback of the 'only Angle condition' approach is that the value of $\theta$ for which we obtain 

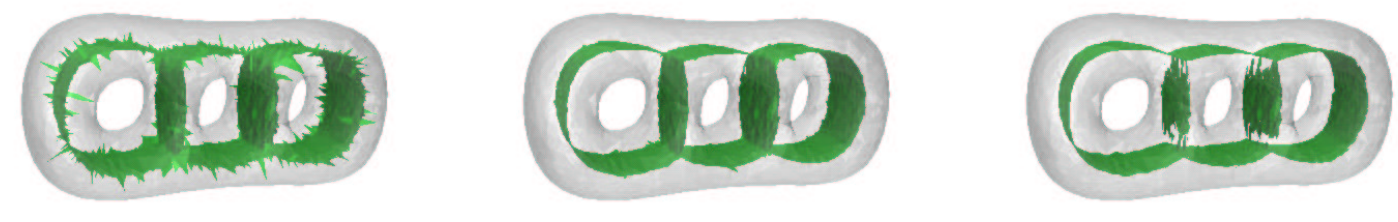

$$
\begin{gathered}
3 \text { HoLE } \\
\theta=3 \text { degrees }
\end{gathered}
$$

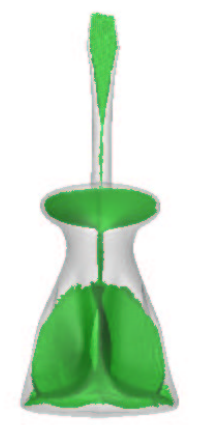

SCREWDRIVER

$\theta=15$ degrees
3 HOLE

$\theta=18$ degrees

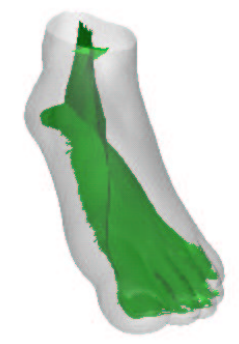

Foot

$\theta=20$ degrees
3 HOLE

$\theta=32$ degrees

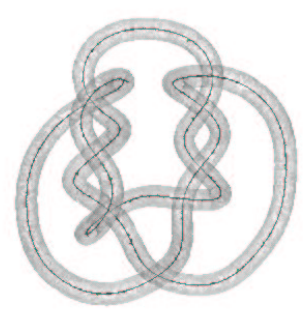

KNOT

$\theta=30$ degrees

Figure 5: Results with 'only Angle condition'.

good approximation differs from sample to sample. It turns out that the required value of $\theta$ gets larger with decreasing sample density. Consequently, we could not find any consistent value for which the approach works for all models we experimented with.

\subsection{Ratio condition}

We have observed that 'only Angle condition' approximates the medial axis well only when we use appropriate $\theta$ for each model. If we fix $\theta$ for all models, some of the medial axis points with medial angle below $\theta$ are not approximated. Consider the medial ball $B$ as shown in Figure 3. From simple geometry of spheres, we get

$$
\|p-q\|=2 \mu \sin \theta
$$

where $\mu=\|m-p\|$ is the radius of $B$. Therefore, if $\theta \gg \varepsilon$, where $\varepsilon$ is the sampling density, we have $\|p-q\| \gg 2 \mu \sin \varepsilon$. Also, it follows from a result in [2] that the radius of the umbrella triangles are only of the order of $\mu \varepsilon$. This means that the length of $p q$ will be much larger than the circumradii of the umbrella triangles.

Therefore, if we compare the length of the Delaunay edges with the circumradii of the umbrella triangles, we can approximate all medial axis points with medial angles only few times larger than $\varepsilon$. Of course, we will not be able to approximate the medial axis points with medial angle less than $\varepsilon$ with this method, but as $\varepsilon$ approaches zero, we get the required convergence. Let $R_{\sigma}$ denote the circumradius of a triangle $\sigma$, refer to Figure 6 . We measure the ratio of the length of a Delaunay edge to the circumradii of the umbrella triangles and consider those that satisfy:

Ratio condition $[\rho]: \min _{\sigma \in \mathrm{U}_{p}} \frac{\|p-q\|}{R_{\sigma}}>\rho$.

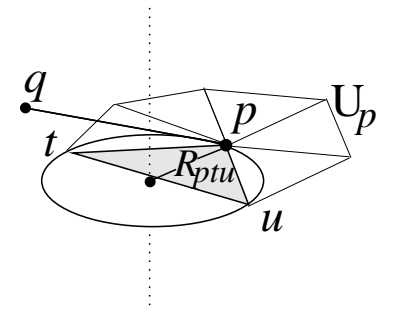

Figure 6: Radius of interest for the Ratio condition $[\rho]$.

\subsubsection{Experiments}

We experimented with the 'only Ratio condition' approach. In this case only those facets are output whose dual Delaunay edges satisfy the Ratio Condition $[\rho]$. No angle condition is considered. As expected, larger value of $\rho$ produces less noisy medial axis, but with 'holes' as shown in the rightmost picture for the FoOT data in Figure 7. On the other hand, smaller $\rho$ produces undesirable spurious 'spikes' as exhibited by the leftmost picture of the FOOT in Figure 7. 


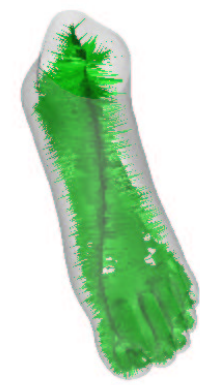

FoоT, $\rho=2$

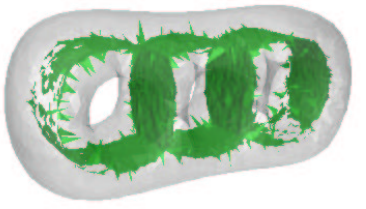

3HoLE, $\rho=2$

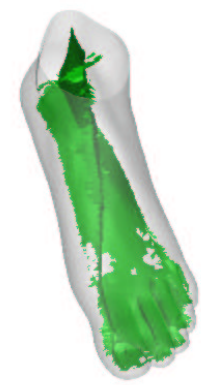

FoOT, $\rho=4$

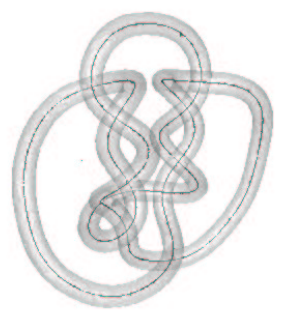

KNOT, $\rho=4$

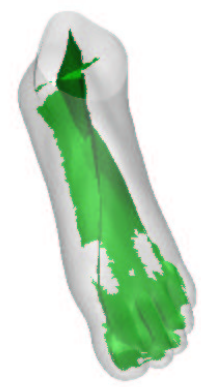

FоOт, $\rho=8$

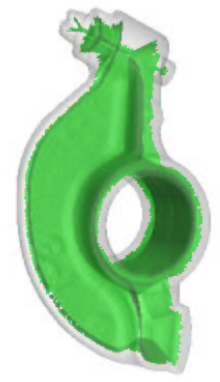

ROCKER, $\rho=6$

Figure 7: Results with 'only Ratio condition'.

In this case also we could not find a value of $\rho$ for which all samples produce good result. See Figure 7 for some examples. When the sample is less dense, a smaller $\rho$ is needed to obtain an approximation as good as possible. 'Only Ratio condition' approach has one more disadvantage. If the sample density is not uniform over the entire surface, one value of $\rho$ cannot capture the medial axis for the entire shape. This is why no value of $\rho$ gave a good result for the FoOT and 3HoLE data though we could find an appropriate $\rho$ for ROCKER and KNOT data which are mostly uniform.

\section{ALGORITHM AND RESULTS}

It is clear from the above experiments that each of Angle and Ratio conditions individually is not sufficient for a good medial axis approximation. Our experiments support that both Angle and Ratio conditions together produce a good approximation to the actual medial axis. We find that the values of $\theta$ and $\rho$ can remain fixed for all tested data sets when we use them together. With a fixed value of $\theta$ all Delaunay edges that lie away from the surface are determined by the Angle condition $[\theta]$ when $\theta$ is sufficiently large. The rest of the Delaunay edges whose duals contribute to the approximate medial axis make small angles with the umbrella triangles but are comparatively larger than their circumradii. They are captured by the Ratio condition $[\rho]$. These two pronged strategy lets both $\theta$ and $\rho$ fixed over different sampling densities. If the density is not high, the Angle condition captures almost all required Delaunay edges. The Ratio condition plays a more significant role when density is high.
Our experiments suggest that $\theta=\frac{\pi}{8}$ and $\rho=8$ are appropriate for all tested data sets. With these two values we enumerate the steps of our algorithm MEDIAL to approximate the medial axis.

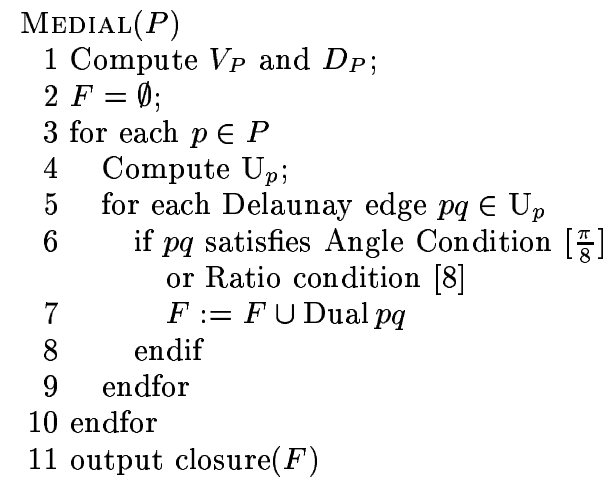

Using the properties of the Voronoi diagram and $\varepsilon$-sampling we prove the following result in a companion paper [13].

THEOREM 1. Let $\boldsymbol{F}_{\varepsilon}$ be the subcomplex computed by $\mathrm{ME}-$ DIAL for an $\varepsilon$-sample of a surface $S \subseteq \mathbb{R}^{3}$ without boundary. We have $\lim _{\varepsilon \rightarrow 0} F_{\varepsilon}=M$, where $M$ is the medial axis of $S$.

Note: The proof of convergence [13] suggests that only ratio condition is sufficient. The anomaly between the theory and practice can be explained as follows. The value of $\varepsilon$ required for theoretical guarantee of converegence is extremely small which often is not the case in practice. The 


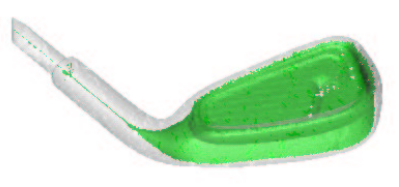

Club

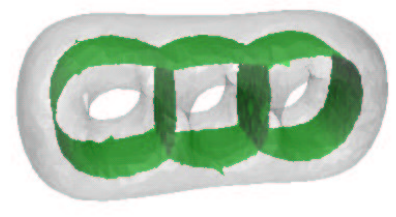

3HoLE

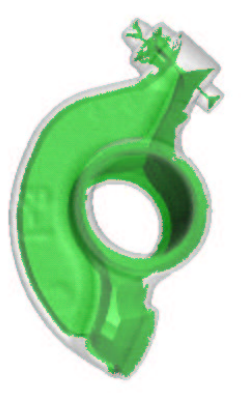

ROCKER

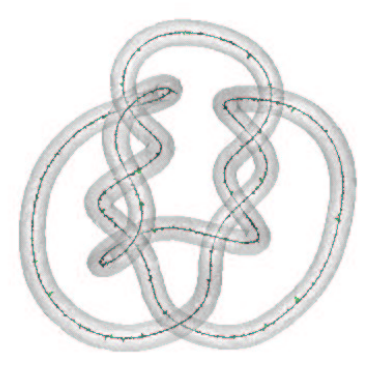

KNOT

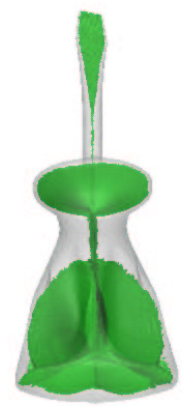

SCREWDRIVER

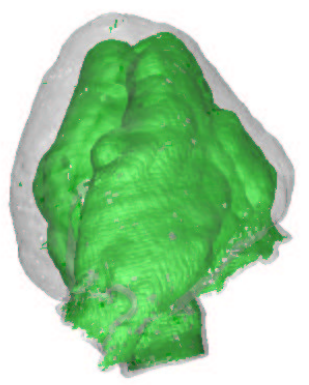

HEART

Figure 8: Medial axis with Medial shown dark shaded.

Angle condition compensates for this shortcoming in practice.

\subsection{Experimental results.}

\subsubsection{Point cloud data.}

Figure 8 and 9 show our result on some example point cloud data sets. In these pictures we show only the 'inner' medial axis that is 'enclosed' by the surface. We computed the 'inner' medial axis as follows. A piecewise linear surface interpolating the sample points is computed with our TIGHT Cocone software [32]. This surface is a subcomplex of the Delaunay triangulation and is guaranteed to be watertight. We output those Voronoi facets computed by MEDIAL that are dual to the Delaunay edges enclosed by the computed surface of Tight Cocone.

The approximation of the medial axis is better where the data is dense. Near high curvature regions, or non-smooth regions where undersampling happens, the approximation contains artifacts. For example, in the ClUB and RockER data, the medial axis is well approximated in most part where the density is high. But, near the handle of the CLUB and top of the RockER, the sampling density suffers due to high curvature and small features. As a result the approximation is poor in these regions.

ROCKER and SCREWDRIVER data are mostly very dense and almost uniform except near some small curvature regions. On the other hand, 3HoLE is relatively sparse data set. In both cases, MEDIAL approximated the medial axis quite well. This shows that MEDIAL is tolerant to different levels of data densities.
In general, the medial axis of a surface is a CW-complex where each cell can be zero, one or two dimensional. In MEDIAL we approximate all cells with two dimensional cells, namely the Voronoi facets. The data set KNOT shows this interesting phenomenon. The one dimensional medial axis in this case has been approximated with very thin Voronoi facets.

The HEART data is extracted as an iso-surface from the intensity field of a volumetric image. The data contains some noise introduced by the iso-surface extraction procedure. MEDIAL could tolerate this noise as the output in Figure 8 suggests.

In Figure 9, we show the two views of the medial axis for FANDISK. The medial axis is well approximated except at the boundaries where it is 'jagged'. The poor approximation at these places is caused by the sharp edges of the surface where it is nonsmooth and thus have inherent problem of undersampling, see [11]. In the FooT data we zoom some places of the toes and the heel. The zoomed region in the toe has undersampling and the medial axis near the boundary has some roughness. However, the heel is well sampled, and the corresponding medial axis boundary is smoother. The Foot data has abrupt density changes in some parts. As a result 'only Ratio condition' did not produce good result for any ratio. MEDIAL produced a good approximation to the medial axis. It shows that MEDIAL is impervious to nonuniformity in data.

\subsubsection{CAD data.}

We experimented with some CAD data sets where the 


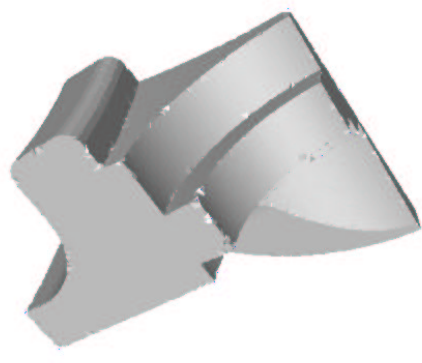

FANDISK

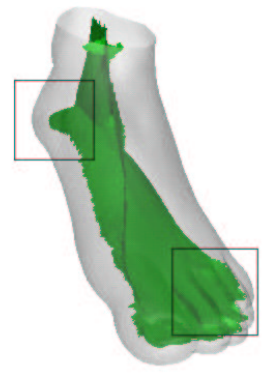

Foot

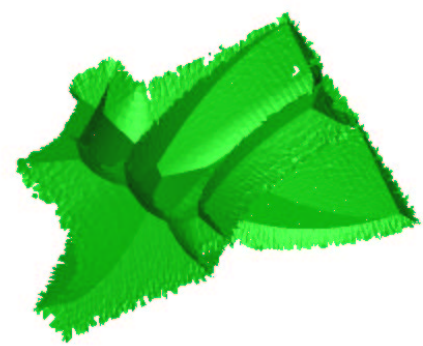

Medial axis

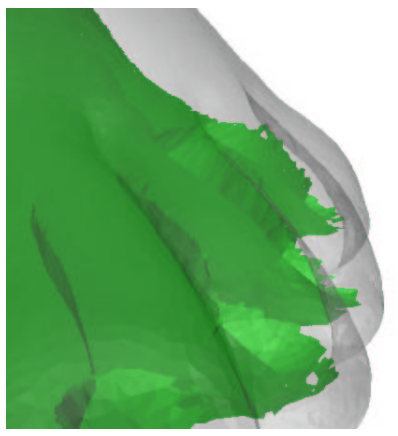

Toes

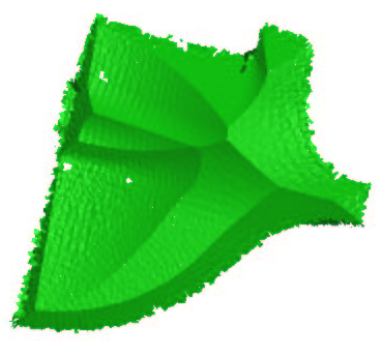

Another view

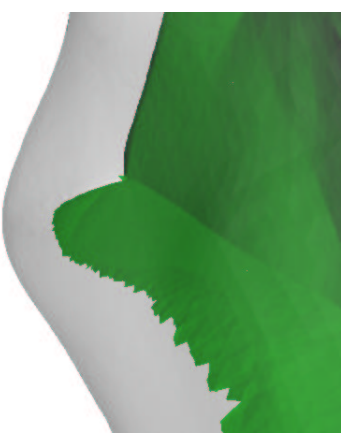

Heel

Figure 9: Computed medial axis of FANDISK and FOOT.

CAD surface was given as input. In this case we know the surface from which we can sample points according to our wish. We sampled the CAD surfaces densely and, in particular, sampled the non-smooth edges and corners. The approximated medial axes are much nicer in these cases, mainly due to the dense sampling of the surfaces which are given as input.

In Figure 10, we show three such CAD examples TAPBOLT, ANCHOR and Engine. The upper row contains the reconstructed surfaces and lower one contains the corresponding medial axes. The medial axis of the TAPBOLT consists of both one and two-dimensional components which are properly captured by MEDIAL. Note that the one-dimensional medail axis along the cylinder is too thin to be visible in this picture, but they actually exist as very tiny Voronoi facets as in KNOT. The ANCHOR shows how MEDIAL computes clean medial axes for complicated shapes.

The sample in ENGINE lie on several connected components. In this case we computed the entire medial axis since 'inner' and 'outer' distinction in this case does not have much meaning.

\subsubsection{Timing}

All of our softwares are written in $\mathrm{C}++$. We used the CGAL library [31] for the Voronoi and Delaunay code and used filtered floating point arithmetic for robust geometric computations. Experiments were conducted on a PC with $933 \mathrm{MHz}$ CPU and 512MB memory. The code was compiled with CGAL2.3 library and g++ compiler with O1 level of optimization. The time for the Delaunay triangulation and filtration are listed in Table 1.

\begin{tabular}{|c|c|c|c|}
\hline object & \# points & $\begin{array}{c}\text { Delaunay } \\
\text { time(sec.) }\end{array}$ & $\begin{array}{c}\text { Filter } \\
\text { time(sec.) }\end{array}$ \\
\hline \hline 3HOLE & 4000 & 2.37 & 0.82 \\
KNOT & 10001 & 8.36 & 2.66 \\
TAPBOLT & 56354 & 333.32 & 9.98 \\
ENGINE & 11361 & 37.0 & 1.82 \\
ANCHOR & 28453 & 87.49 & 5.14 \\
FANDISK & 16475 & 13.4 & 3.0 \\
CLUB & 16865 & 18.81 & 3.06 \\
FOOT & 20021 & 13.24 & 3.71 \\
SCREWDRIVER & 27152 & 51.87 & 5.36 \\
HEART & 37912 & 32.49 & 6.58 \\
ROCKER & 40177 & 74.54 & 8.25 \\
\hline
\end{tabular}

Table 1: Time data.

\subsubsection{Comparisons}

We comment on two most competitive approaches for approximating the medial axis from point samples, one by Attali et. al $[4,5]$ and the other by Amenta et. al [3].

Attali and Montanvert [5] first proposed a filtration method depending only on an angle condition similar to ours though not same. We have already seen that 'only Angle condition' does not produce good approximations with a fixed angle threshold. This method depends heavily on the data density. Later Attali and Lachaud [4] proposed two criteria for the filtration.

They compute the Voronoi diagram and extract the com- 

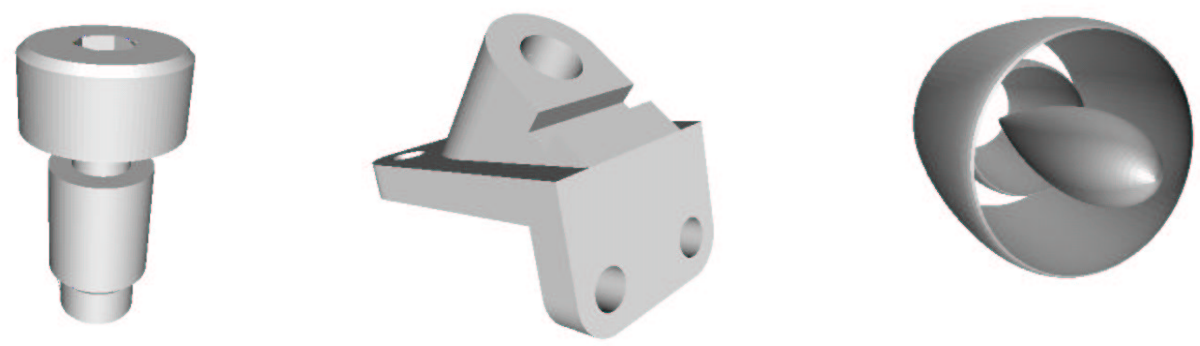

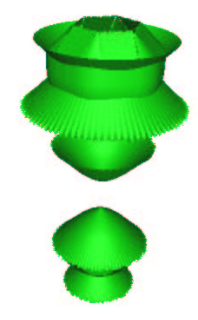

TAPBOLT

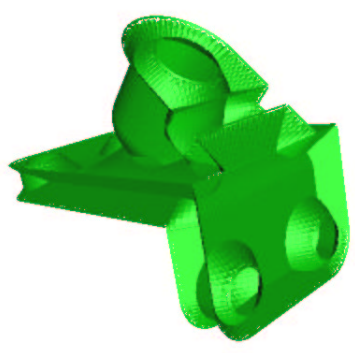

ANCHOR

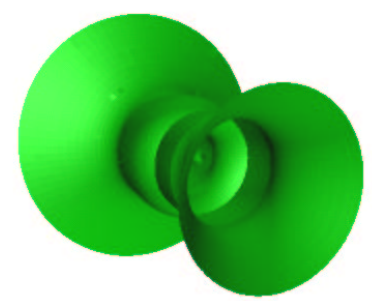

ENGINE

Figure 10: Medial axis with MEDIAL of some CAD models. The surfaces and the corresponding medial axes are shown in upper and lower row respectively.

plex enclosed by the surface. Next, they approximate the radius $\mu$ of the medial ball centered at a medial axis point $m$ near a Voronoi vertex $v$ by computing the radius of the Delaunay ball centered at $v$. Also, they approximate the medial angle $\theta$ for $m$ by computing the maximum angle made by two Voronoi edges incident to $v$. They only retain those Voronoi vertices and their incident edges and facets for which the computed value of $\mu$ and $\theta$ are more than certain thresholds.

Clearly, this method depends on scaling as $\mu$ is computed in absolute terms. Even the angle $\theta$, as argued earlier, requires a sampling dependent threshold. This is why this method needed different thresholds for the example models considered in [4]. Our method differs in two fundamental ways. First, all comparisons are relative to the umbrella triangles in our method which make it scale independent and immune to varying densities. Secondly, instead of using an 'and' between two conditions, we use 'or'. This is also necessary to achieve immunity against density variations. We have already shown that 'only Angle condition' cannot find a common threshold and requiring another condition conjunctively makes the filtration even stricter.

The Power Crust algorithm by Amenta et. al [3] produces an approximation to the medial axis as a dual shape, which is called the Power Shape. This algorithm first computes the poles from the Voronoi diagram of the sample points. Next, a power diagram of the Delaunay balls centered at the poles is computed. The dual weighted Delaunay triangulation with the poles as vertices, called Power Shape, is output as the approximate medial axis. This method uses two
Voronoi diagram computations, one to compute the poles and another to compute the power diagram, as opposed to our approach which approximates the medial axis straight from the Voronoi diagram. Worse, the Power Shape produces sometimes too many 'spikes' as in the Foot, HEART data and ANCHOR data, see Figure 11. The authors propose to clean up the noisy medial axis using criteria similar to Attali et. al [4] which suffers from the scale and density dependencies as we pointed out earlier.

\section{SIMPLIFICATION}

Mesh simplification that reduces the number of elements is widely used in graphics and modeling so that further processing can be done efficiently. Of course, the major concern in simplification is preserving the shape while still reducing the mesh size. First, we experimented with a strategy of simplifying the medial axis that operates at the sample level rather than directly on the medial axis. This has the advantage that one does not have to worry about which parts of the medial axis need to be deleted so that its shape is still preserved. Instead of collapsing edges that is used in usual mesh simplification we first decimate the samples and then construct their medial axis with MEDIAL. In [12] we showed how a sample can be decimated while still keeping it dense enough for surface reconstruction. This means all high level feature information are preserved by the sample decimation.

Figure 12 shows the approximate medial axes computed out of these samples. The medial axes become coarser as the samples are decimated.

In a second experiment we carried out the simplification 


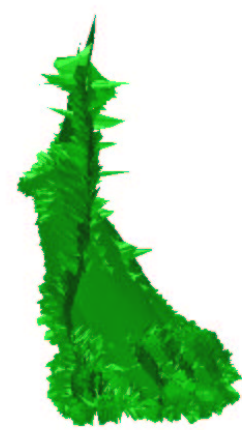

FoOT

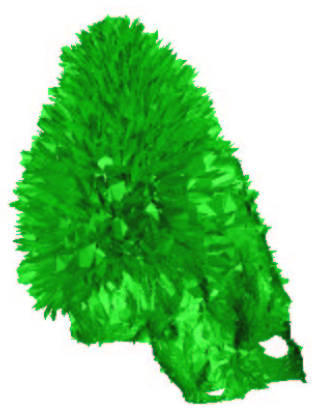

HEART

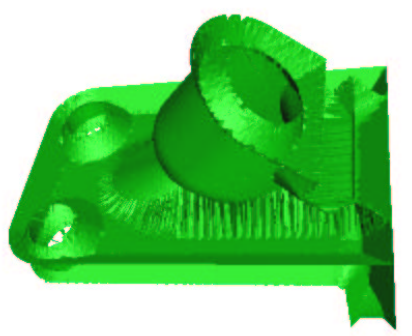

ANCHOR

Figure 11: Medial axis approximation with the Power Shape of [3] generates too many spikes.

without any sample decimation. Medial axis is sensitive to small changes in shape. Since a finite sample cannot capture the complete information of a surface, the uncertainty in surface definition shows up in the medial axis as 'spikes' that abut from a more stable part. MEDIAL already captures only this stable part of the medial axis. But, even within the 'stable' parts there is a hierarchy of 'stability'. Some parts are more stable than others. One can associate this stabilty with the angle $\theta$ and the ratio $\rho$. In Figure 13 we show how larger values of $\theta$ and $\rho$ would capture the subset with higher 'stability'.

\section{CONCLUSIONS}

In this paper we proposed and experimented with an algorithm that approximates the medial axis from the Voronoi diagram of a set of sample points. Unlike previous approaches, this algorithm is scale and density independent. It is known that a small change in shape can cause the medial axis change considerably. This leads to unwanted 'spikes' in the medial axis which abut from a more 'stable' part. A finite sample cannot capture a shape completely and this small uncertainty in shape definition shows up in the medial axis approximation through the noisy spikes. The challenge was to remove this uncertainty from the medial axis approximation in a manner impervious to scale and density. Our empirical results suggest that our algorithm achieves this goal.

In many applications it is useful to have a simplification of the medial axis. We showed a method to simplify the medial axis in terms of the number of elements. If the simplification is to be in terms of its stability, we can use larger values for the filter conditions. Is there way to determine the parameter values automatically that bring up the hierarchy of the medial axis in terms of stabilty? More investigations are necessary to answer this question.

\section{Acknowledgement}

We thank Dominique Attali for sending the HEART data.

\section{REFERENCES}

[1] N. Amenta and M. Bern. Surface reconstruction by Voronoi filtering. Discr. Comput. Geom. 22 (1999), 481-504.
[2] N. Amenta, S. Choi, T. K. Dey and N. Leekha. A simple algorithm for homeomorphic surface reconstruction. Internat. J. Comput. Geom. Applications, 12 (2002), 125-141.

[3] N. Amenta, S. Choi and R. K. Kolluri. The power crust. Proc. Solid Modeling '01, (2001), 249-260.

[4] D. Attali and J.-O. Lachaud. Delaunay conforming iso-surface, skeleton extraction and noise removal. Comput. Geom. : Theory Appl. 19 (2001), 175-189.

[5] D. Attali and A. Montanvert. Computing and simplifying 2D and 3D continuous skeletons. Computer Vision and Image Understanding 67 (1997), 261-273.

[6] S. Bouix and K. Siddiqi. Divergence-based medial surfaces. Proc. European Conference on Computer Vision, 2000.

[7] J. D. Boissonnat and F. Cazals. Smooth surface reconstruction via natural neighbor interpolation of distance functions. Proc. 16th. ACM Sympos. Comput. Geom., (2000), 223-232.

[8] J. W. Brandt. Convergence and continuity criteria for discrete approximation of the continuous planar skeletons. CVGIP: Image Understanding 59 (1994), 116-124.

[9] J. W. Brandt and V. R. Algazi. Continuous skeleton computation by Voronoi diagram. Comput. Vision, Graphics, Image Process. 55 (1992), 329-338.

[10] T. Culver, J. Keyser and D. Manocha. Accurate computation of the medial axis of a polyhedron. Solid Modeling '99, (1999), 179-190.

[11] T. K. Dey and J. Giesen. Detecting undersampling in surface reconstruction. Proc. 17th Ann. Sympos. Comput. Geom. (2001), 257-263.

[12] T. K. Dey, J. Giesen and J. Hudson. Decimating samples for mesh simplification. Proc. 13th Canadian Conf. Comput. Geom. (2001), 85-88.

[13] T. K. Dey and W. Zhao. Approximating the medial axis from the Voronoi diagram with a convergence guarantee. Algorithmica, to appear. http://www.cis.ohiostate.edu/ tamaldey/paper/medial.pdf.

[14] H. Edelsbrunner. Shape reconstruction with Delaunay complex. Proc. Sympos. Latin Amenrican Theoret. Inform. 1998, Campinas, Brazil, Springer-Verlag 


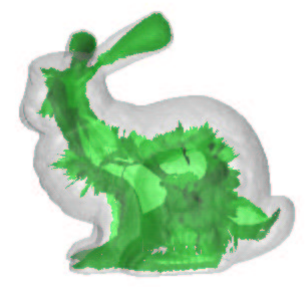

BUNNY

$\sim 36 \mathrm{~K}$ sample points, $\sim 30 \mathrm{~K}$ medial facets

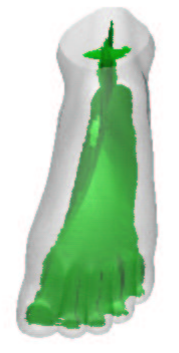

FoOT

$\sim 20 \mathrm{~K}$ sample points

$\sim 27 \mathrm{~K}$ medial facets

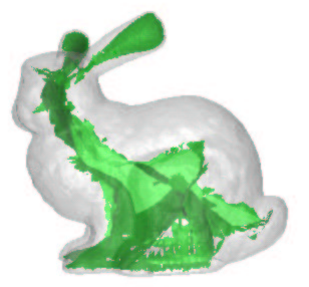

BUNNY

$\sim 11 \mathrm{~K}$ sample points

$\sim 11 \mathrm{~K}$ medial facets

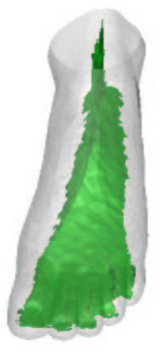

FoOT

$\sim 3 \mathrm{~K}$ sample points $\sim 4 \mathrm{~K}$ medial facets

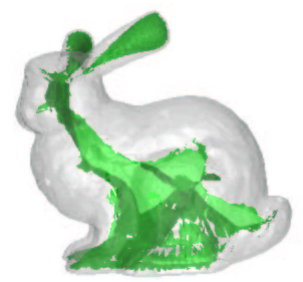

BUNNY

$\sim 7 \mathrm{~K}$ sample points

$\sim 8 \mathrm{~K}$ medial facets

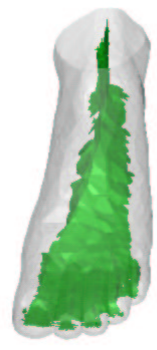

FooT

$\sim 2 \mathrm{~K}$ sample points

$\sim 2 \mathrm{~K}$ medial facets

Figure 12: Medial axis at different level of sampling density for BUNNY and FoOT.

Lecture Notes 1380, 119-132.

[15] H. Edelsbrunner and E. P. Mücke. Three-dimensional alpha shapes. ACM Trans. Graphics 13 (1994), 43-72.

[16] M. Etzion and A. Rappoport. Computing Voronoi skeletons of a 3D polyhedron by space subdivision. Tech. Report, Hebrew University, 1999.

[17] P. J. Giblin and B. B. Kimia. A formal classification of 3D medial axis points and their local geometry. Proc. Computer Vision and Pattern Recognition (CVPR), 2000.

[18] L. Guibas, R. Holleman and L. E. Kavraki. A probabilistic roadmap planner for flexible objects with a workspace medial axis based sampling approach. Proc. IEEE/RSJ Intl. Conf. Intelligent Robots and Systems, 1999.

[19] H. N. Gursoy and N. M. Patrikalakis. Automated interrogation and adaptive subdivision of shape using medial axis transform. Advances in Engineering Software 13 (1991), 287-302.

[20] C. Hoffman. How to construct the skeleton of CSG objects. Proc. Fourth IMA Conf., The Mathematics of Surfaces, A. Bowyer and J. Davenport Eds., University of Bath, UK, September 1990.

[21] P. Hubbard. Approximating polyhedra with spheres for time critical collision detection. ACM Trans. Graphics 15 (1996), 179-210.
[22] C. Niblak, P. Gibbons and D. Capson. Generating skeletons and centerlines from the distance transform. CVGIP: Graphical Models and Image Processing $\mathbf{5 4}$ (1992), 420-437.

[23] R. L. Ogniewicz. Skeleton-space: A multiscale shape description combining region and boundary information. Proc. Computer Vision and Pattern Recognition, (1994), 746-751.

[24] D. Sheehy, C. Armstrong and D. Robinson. Shape description by medial axis construction. IEEE Trans. Visualization and Computer Graphics 2 (1996), 62-72.

[25] A. Sheffer, M. Etzion, A. Rappoport and M. Bercovier. Hexahedral mesh generation using the embedded Voronoi graph. Engineering Comput. 15 (1999), 248-262.

[26] E. C. Sherbrooke, N. M. Patrikalakis and E. Brisson. An algorithm for the medial axis transform of $3 \mathrm{D}$ polyhedral solids. IEEE Trans. Vis. Comput. Graphics 2 (1996), 44-61.

[27] D. Storti, G. Turkiyyah, M. Ganter, C. Lim and D. Stal. Skeleton-based modeling operations on solids. Solid Modeling '97' (1997), 141-154.

[28] G. M. Turkiyyah, D. W. Storti, M. Ganter, H. Chen and M. Vimawala. An accelerated triangulation method for computing the skeletons of free-form solid models. Computer Aided Design 29 (1997), 5-19.

[29] M. Teichman and S. Teller. Assisted articulation of 

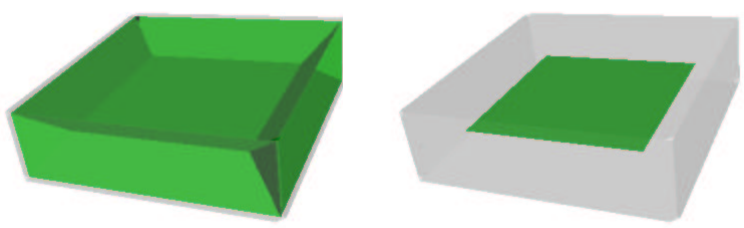

Box

$\rho=8, \theta=22.5$

Box

$\rho=12, \theta=50$
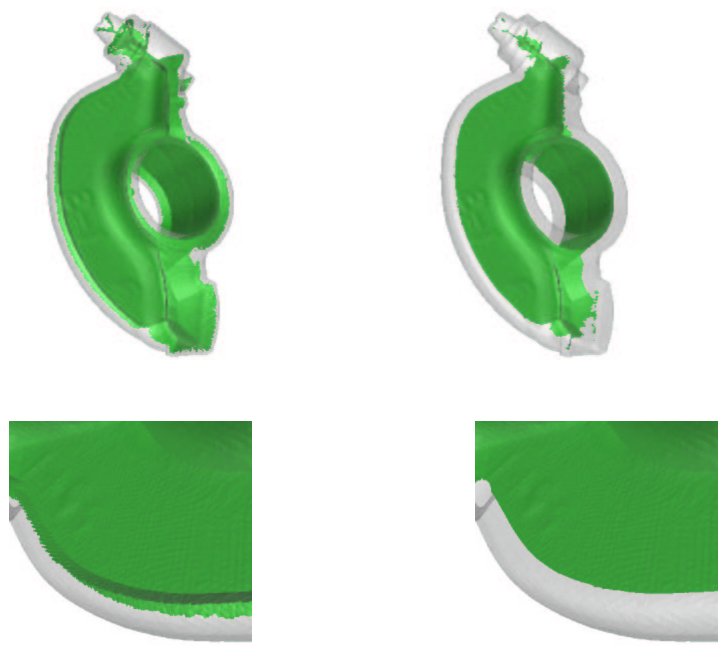

ROCKER

$\rho=8, \theta=22.5$

ROCKER

$\rho=12, \theta=50$

Figure 13: Larger parameters capture more stable parts of the medial axis. The zoomed figure shows how the medial axis of ROCKER got cleaned near the edge with larger parameters.

closed polygonal models. Proc. 9th Eurographics Workshop on Animation and Simulation, 1998.

[30] F.-E. Wolter. Cut locus \& medial axis in global shape interrogation \& representation. MIT Design Laboratory Memorandum 92-2, 1992.

[31] www.cgal.org.

[32] www.cis.ohio-state.edu/ tamaldey/cocone.html. 